\title{
Incidence of remission and relapse of proteinuria, end-stage kidney disease, mortality, and major outcomes in primary nephrotic syndrome: the Japan Nephrotic Syndrome Cohort Study (JNSCS)
}

\author{
Ryohei Yamamoto ${ }^{1,2}\left(\right.$ Enyu Imai $^{3} \cdot$ Shoichi Maruyama ${ }^{4} \cdot$ Hitoshi Yokoyama $^{5} \cdot$ Hitoshi Sugiyama $^{6} \cdot$ Kosaku Nitta $^{7}$. \\ Tatsuo Tsukamoto $^{8}$. Shunya Uchida ${ }^{9} \cdot$ Asami Takeda $^{10} \cdot$ Toshinobu Sato $^{11} \cdot$ Takashi Wada $^{12} \cdot$ Hiroki Hayashi $^{13}$. \\ Yasuhiro Akai ${ }^{14}$. Megumu Fukunaga ${ }^{15} \cdot$ Kazuhiko Tsuruya $^{16} \cdot$ Kosuke Masutani $^{17}$. Tsuneo Konta ${ }^{18}$. Tatsuya Shoji ${ }^{19}$. \\ Takeyuki Hiramatsu ${ }^{20}$. Shunsuke Goto ${ }^{21}$. Hirofumi Tamai ${ }^{22}$. Saori Nishio ${ }^{23}$ - Arimasa Shirasaki $^{24} \cdot$ Kojiro Nagai $^{25}$. \\ Kunihiro Yamagata ${ }^{26} \cdot$ Hajime Hasegawa $^{27} \cdot$ Hideo Yasuda $^{28}$. Shizunori Ichida ${ }^{29} \cdot$ Tomohiko Naruse $^{30}$.

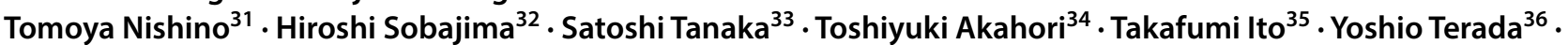 \\ Ritsuko Katafuchi ${ }^{37}$. Shouichi Fujimoto ${ }^{38}$. Hirokazu Okada ${ }^{39}$. Eiji Ishimura ${ }^{40}$. Junichiro J. Kazama ${ }^{41}$. \\ Keiju Hiromura ${ }^{42} \cdot$ Tetsushi Mimura $^{43} \cdot$ Satoshi Suzuki ${ }^{44} \cdot$ Yosuke Saka $^{45} \cdot$ Tadashi Sofue $^{46} \cdot$ Yusuke Suzuki $^{47}$. \\ Yugo Shibagaki ${ }^{48} \cdot$ Kiyoki Kitagawa $^{49} \cdot$ Kunio Morozumi $^{50} \cdot$ Yoshiro Fujita $^{51} \cdot$ Makoto Mizutani $^{52}$. \\ Takashi Shigematsu ${ }^{53} \cdot$ Naoki Kashihara $^{54} \cdot$ Hiroshi Sato $^{55} \cdot$ Seiichi Matsuo ${ }^{4}$. Ichiei Narita ${ }^{56}$. Yoshitaka Isaka ${ }^{2}$
}

Received: 24 December 2019 / Accepted: 18 February 2020 / Published online: 7 March 2020 (c) The Author(s) 2020

\begin{abstract}
Background Despite recent advances in immunosuppressive therapy for patients with primary nephrotic syndrome, its effectiveness and safety have not been fully studied in recent nationwide real-world clinical data in Japan.

Methods A 5-year cohort study, the Japan Nephrotic Syndrome Cohort Study, enrolled 374 patients with primary nephrotic syndrome in 55 hospitals in Japan, including 155, 148, 38, and 33 patients with minimal change disease (MCD), membranous nephropathy (MN), focal segmental glomerulosclerosis (FSGS), and other glomerulonephritides, respectively. The incidence rates of remission and relapse of proteinuria, 50\% and 100\% increases in serum creatinine, end-stage kidney disease (ESKD), all-cause mortality, and other major adverse outcomes were compared among glomerulonephritides using the Log-rank test. Incidence of hospitalization for infection, the most common cause of mortality, was compared using a multivariable-adjusted Cox proportional hazard model.

Results Immunosuppressive therapy was administered in 339 (90.6\%) patients. The cumulative probabilities of complete remission within 3 years of the baseline visit was $\geq 0.75$ in patients with MCD, MN, and FSGS $(0.95$, 0.77, and 0.79, respectively). Diabetes was the most common adverse events associated with immunosuppressive therapy (incidence rate, 71.0 per 1000 person-years). All-cause mortality (15.6 per 1000 person-years), mainly infection-related mortality (47.8\%), was more common than ESKD (8.9 per 1000 person-years), especially in patients with MCD and MN. MCD was significantly associated with hospitalization for infection than MN.

Conclusions Patients with MCD and MN had a higher mortality, especially infection-related mortality, than ESKD. Nephrologists should pay more attention to infections in patients with primary nephrotic syndrome.
\end{abstract}

Keywords Primary nephrotic syndrome $\cdot$ Cohort study $\cdot$ Mortality $\cdot$ End-stage kidney disease $\cdot$ Diabetes $\cdot$ Infection

Electronic supplementary material The online version of this article (https://doi.org/10.1007/s10157-020-01864-1) contains supplementary material, which is available to authorized users.

Ryohei Yamamoto

yamamoto@hacc.osaka-u.ac.jp

Extended author information available on the last page of the article

\section{Introduction}

Nephrotic syndrome is characterized by massive proteinuria, edema, and hypoalbuminuria [1]. Epidemiological studies have shown that patients with nephrotic syndrome are vulnerable to a wide variety of adverse events: end-stage kidney disease (ESKD) [2-4], thromboembolism [5], infection 
[6], malignancy [7], cardiovascular disease (CVD) [8], and all-cause mortality [9]. Primary nephrotic syndrome is the major cause of nephrotic syndrome diagnosed using kidney biopsy, including mainly minimal change disease (MCD), membranous nephropathy (MN), and focal segmental glomerulosclerosis (FSGS) [10]. A systematic review reported that incidences rates of MCD, MN, and FSGS were $0.2-0.8$, $0.3-1.4$, and $0.2-1.1$ per 100,000 person-years, respectively [11].

Immunosuppressive therapy is the main treatment modality for patients with primary nephrotic syndrome as suggested by the clinical guidelines of primary nephrotic syndromes [12, 13]. Systematic reviews of randomized controlled trials on immunosuppressive therapy in patients with $\mathrm{MN}$, the most extensively studied glomerulonephritis in primary nephrotic syndrome, clarified that some immunosuppressive drugs reduced all-cause mortality and risk of ESKD, although the number of trials with a high-quality design was relatively small and most trials did not have adequate follow-up and enough power to assess the prespecified definite outcomes $[14,15]$. These systematic reviews also suggested that the drugs were associated with substantial toxicity leading to withdrawals or hospitalization. Their results potentially underestimated the toxicity of immunosuppressive therapy in the real world because patients with a higher risk of toxicity, such as elderly patients, are often excluded in randomized trials [16]. To establish the treatment strategy that has a high effectiveness and low risk of adverse effects, an observational study using real-world data, including patients with a high risk of toxicity from therapeutic interventions, is essential.

The aim of the present cohort study, the Japan Nephrotic Syndrome Cohort Study (JNSCS) [17], was to clarify the incidence of major clinical outcomes in 374 patients with primary nephrotic syndrome during the 5-year follow-up period. The outcomes of interest were remission and relapse of proteinuria, deterioration in kidney function $(50 \%$ and $100 \%$ increases in serum creatinine level and ESKD), CVD, all-cause mortality, and other adverse events associated with immunosuppressive therapy, including infection, diabetes, arteriovenous thrombosis, aseptic osteonecrosis, and peptic ulcers. The results of the present study provide pivotal information to determine the clinical goals of the treatments for primary nephrotic syndrome.

\section{Materials and methods}

\section{Participants}

The JNSCS is a 5-year multicenter cohort study of primary nephrotic syndrome to clarify the incidence rates of major clinical outcomes and assess the effectiveness of immunosuppressive therapy in Japan. Details of the study design was described elsewhere [17]. Briefly, 455 nephrotic patients were registered in the JNSCS, who were diagnosed with primary nephrotic syndrome using kidney biopsy during the entry period between January 2009 and December 2010 in 56 hospitals (Fig. 1). The diagnosis of primary nephrotic syndrome was based on the clinical and histopathological characteristics [18]. Nephrotic patients with minor glomerular abnormalities by light microscopy was diagnosed as MCD. The diagnosis of MN was made by the detection of granular deposits of mainly $\mathrm{IgG}$ along the glomerular capillary walls by immunofluorescence microscopy with or without thickening of the glomerular capillary wall by light microscopy. FSGS included five variants: collapsing, tip, cellular, perihilar, and not-otherwise specified (NOS) variants [19]. After excluding 81 patients with no kidney biopsy $(N=20)$, kidney biopsy before or after the entry period $(N=32)$, no history of nephrotic syndrome $(N=1)$, diagnosis of secondary nephrotic syndrome $(N=13)$, sclerosing glomerulonephritis with unknown etiology $(N=1)$, incomplete informed consent $(N=7)$, duplicate registrations $(N=3)$ and unknown reason $(N=4), 374$ patients with primary nephrotic syndrome in 55 hospitals were finally enrolled in JNSCS, including those with MCD $(N=155), \mathrm{MN}(N=148)$, FSGS $(N=38)$, IgA nephropathy $(N=15)$, membranoproliferative glomerulonephritis $(N=9)$, mesangial proliferative glomerulonephritis $(N=5)$, endocapillary proliferative glomerulonephritis $(N=2)$, and crescentic glomerulonephritis $(N=2)$. Because of the small number of patients with glomerulonephritides except those with MCD, MN, and FSGS, the patients were classified into four groups of glomerulonephritides: MCD, MN, FSGS, and other glomerulonephritides.

The study protocol of JNSCS was approved by the ethics committee of Osaka University Hospital (approval number 17035-4) and the institutional review board of each participating hospital. All procedures performed in the present study were in accordance with the World Medical Association Declaration of Helsinki.

\section{Measurements}

The clinical characteristics at the kidney biopsy and, if immunosuppressive therapy was administered, those at initiating immunosuppressive therapy were collected in JNSCS, including age, sex, body mass index, systolic and diastolic blood pressure, 24 -h urinary protein, urinary protein-to-creatinine ratio, serum concentration of creatinine, albumin, and total cholesterol, hemoglobin A1c, and use of reninangiotensin system (RAS) blockers, statins, and antidiabetic drugs. To calculate the estimated glomerular filtration rate (eGFR) in adult patients aged 18 years or older, the Japanese equation was used: eGFR $=194 \times$ age $(\text { year })^{-0.287} \times$ serum 
Fig. 1 Flow diagram of patients in the Japan Nephrotic Syndrome Cohort Study (JNSCS). *Including two patients who were diagnosed with MCD at the first kidney biopsy but re-diagnosed with FSGS (NOS variant) at the second biopsy 33 and 1344 days after the first biopsy

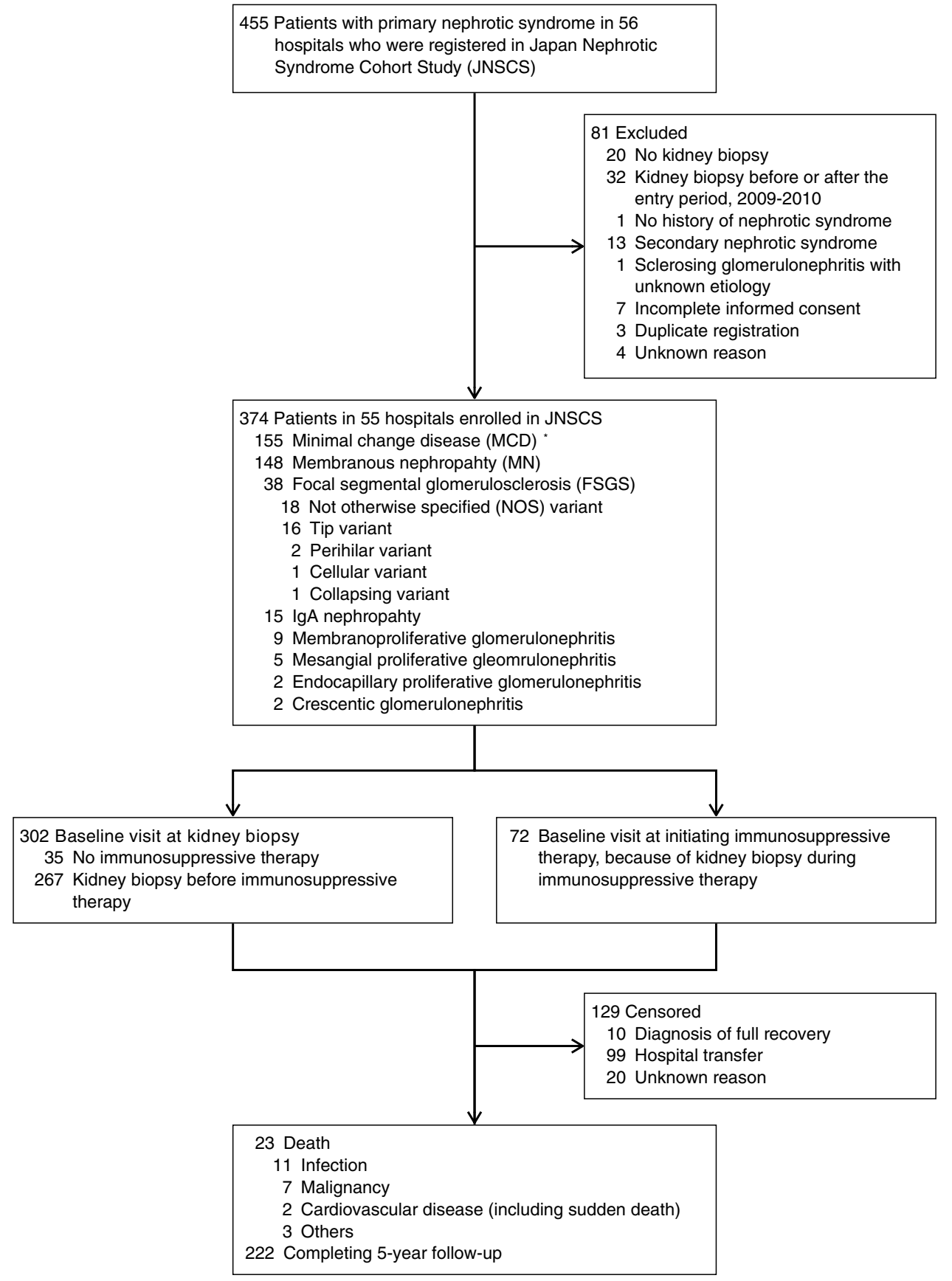

creatinine $(\mathrm{mg} / \mathrm{dL})^{-1.094} \times 0.739$ (if female) [20]. As a measure of the baseline urinary protein, 24-h urinary protein was preferred. Urinary protein-to-creatinine ratio was alternatively used only in patients with missing values of the baseline 24-h urinary protein. In the present study, the baseline visit was set at the kidney biopsy or the first date of immunosuppressive therapy, whichever came first.

The outcome measures of interest in the present study consisted of the time to remission and relapse of proteinuria; $50 \%$ and $100 \%$ irreversible increases in serum creatinine level; ESKD requiring kidney replacement therapy; use of antidiabetic drugs; hospitalization for infection, CVD, and arteriovenous thrombosis; diagnosis of malignancy, aseptic osteonecrosis, and peptic ulcer; and all-cause mortality. Remission of proteinuria was categorized into complete remission, incomplete remission type 1 , and incomplete remission type 2 ; complete remission was defined as $24-\mathrm{h}$ urinary protein of $<0.3 \mathrm{~g} /$ day or urinary protein-to-creatinine ratio of $<0.3 \mathrm{~g} / \mathrm{gCr}$; incomplete remission type 1 was defined as $24-\mathrm{h}$ urinary protein of $<1.0 \mathrm{~g} /$ day or urinary protein-to-creatinine ratio of $<1.0 \mathrm{~g} / \mathrm{gCr}$; incomplete remission type 2 was defined as 24 -h urinary protein of $<3.5 \mathrm{~g} /$ day or 
urinary protein-to-creatinine ratio of $<3.5 \mathrm{~g} / \mathrm{gCr}[2,13]$. Relapse of proteinuria was defined as 24-h urinary protein of $\geq 1.0 \mathrm{~g} /$ day, urinary protein-to-creatinine ratio of $\geq 1.0 \mathrm{~g} /$ $\mathrm{gCr}$, and/or $2+$ or more of positive dipstick tests for urinary protein continued two times or more in patients with complete remission [13]. CVD included heart disease, stroke, peripheral arterial disease, and sudden death. In patients followed up for more than 5 years, the end of the follow-up was set at 5 years after the baseline visit of each patient. Patients who died were regarded as censored, except in the analyses of all-cause mortality (Supplementary Fig. 1A and B).

\section{Statistical analyses}

Baseline clinical characteristics among the four groups of glomerulonephritides were compared using the chi-square test, Fisher's exact test, ANOVA, or Kruskal-Wallis test, as appropriate.

To compare the incidence rates of each outcome among the four groups of glomerulonephritides, their cumulative probabilities were estimated using the Kaplan-Meier method and compared using the Log-rank test. The cumulative probabilities of complete remission, incomplete remission type 1 , and incomplete remission type 2 were calculated in 292,367 , and 370 patients with baseline urinary protein of $\geq 0.3, \geq 1.0$, and $\geq 3.5 \mathrm{~g} / \mathrm{day}$ (or $\mathrm{g} / \mathrm{gCr}$ ), respectively (Supplementary Fig. 1A). To calculate those of relapse of proteinuria after complete remission, 290 patients who achieved complete remission and were followed up thereafter were included. After excluding 16 patients with the baseline use of diabetic drugs, 358 patients with no baseline use of antidiabetic drugs were included for calculation of the cumulative probability of use of diabetic drugs (Supplementary Fig. 1B). The incidence rate of each outcome was calculated based on the Poisson distribution and expressed as the number of events per 1000 person-years.

Because infection was the leading cause of mortality, the incidence of hospitalization for infection was compared among the four groups of glomerulonephritides using unadjusted and multivariable-adjusted Cox proportional hazards models. The proportional hazards assumption for covariates was checked using Schoenfeld residuals. Because the proportional hazards assumption of sex was violated, all multivariable-adjusted Cox proportional hazards models were stratified according to sex to control its potential confounding effect. Multivariable-adjusted model 1 included age as covariates. Models 2 and 3 included serum creatinine and urinary protein as covariates in an additive manner.

Normally distributed continuous variables are expressed as mean \pm standard deviation, and non-normally distributed continuous variables as median (interquartile range). Categorical variables are expressed as numbers (proportions). $P<0.05$ was considered statistically significant. All statistical analyses were performed using $\mathrm{R}$ version 3.6.0 (The R Foundation for Statistical Computing, https://www.rproject.org/).

\section{Results}

The clinical characteristics of 155 (41.4\%), 148 (39.6\%), 38 (10.2\%), and 33 (8.8\%) patients with MCD, MN, FSGS, and others, respectively, are listed in Table 1 . The baseline visit was set at the beginning of immunosuppressive therapy in 45 (29.0\%), $14(9.5 \%), 9(23.7 \%)$, and $4(12.1 \%)$ patients with MCD, MN, FSGS, and others, respectively, because they underwent kidney biopsy after initiating immunosuppressive therapy, whereas it was set at the date of kidney biopsy in the remaining patients. At their baseline visits, significant differences among the four groups were observed in terms of the age, body mass index, systolic and diastolic blood pressure, urinary protein, serum creatinine, eGFR, serum albumin, serum total cholesterol, and use of RAS blockers, statin, and antidiabetic drugs $(P<0.05)$. Patients with MCD were likely to have received immunosuppressive therapy before kidney biopsy and have a younger age, higher levels of body mass index, eGFR, and serum total cholesterol, lower levels of blood pressure, serum creatinine and serum albumin, and lower proportion of use of RAS blockers, compared with that in patients with MN, FSGS, and others.

The majority of patients received immunosuppressive therapy within a median (interquartile range) of $3(-4,7)$, $10(4,24), 6(0,14)$, and $10(4,14)$ days of kidney biopsy in 153 (98.7\%), 127 (85.8\%), 35 (92.1\%), and 24 (72.7\%) patients with MCD, MN, FSGS, and others, respectively (Table 1). Almost all patients received prednisolone within 24 months of immunosuppressive therapy $(98.7 \%, 95.2 \%$, $100.0 \%$, and $100.0 \%$ in MCD, MN, FSGS, and others). One-third of the patients with MCD additionally received intravenous methylprednisolone (31.6\%) and cyclosporine (35.5\%) within 24 months of immunosuppressive therapy. In patients with MN and FSGS, cyclosporine (56.5\% and $62.9 \%$, respectively) was much more common than intravenous methylprednisolone ( $21.0 \%$ and $31.4 \%$, respectively). In contrast, a half of patients with other glomerulonephritides received intravenous methylprednisolone (50.0\%), followed by cyclosporine (27.3\%). Other immunosuppressive drugs were rarely used, except mizoribine in patients with MN (18.5\%).

The cumulative probabilities of remission and relapse of proteinuria during the median follow-up period of 5.0 years (interquartile range $3.2-5.0$ ) are described in Fig. 2a-d. Within one year of the baseline visit, urinary protein decreased below the nephrotic range of proteinuria indicating incomplete remission type 2, in approximately $80 \%$ or more of the nephrotic patients at the baseline visit 
Table 1 Clinical characteristics of 374 patients with primary nephrotic syndrome

\begin{tabular}{|c|c|c|c|c|}
\hline & MCD & MN & FSGS & Others \\
\hline$N$ & 155 & 148 & 38 & 33 \\
\hline \multicolumn{5}{|l|}{ Baseline visit, $N(\%)^{* a}$} \\
\hline Kidney biopsy & $110(71.0)$ & $134(90.5)$ & $29(76.3)$ & $29(87.9)$ \\
\hline Immunosuppressive therapy & $45(29.0)$ & $14(9.5)$ & $9(23.7)$ & $4(12.1)$ \\
\hline \multicolumn{5}{|l|}{ Clinical characteristics at baseline visit } \\
\hline Age (year) ${ }^{*}$ & $41(26,61)$ & $66(59,74)$ & $62(29,73)$ & $58(46,71)$ \\
\hline$<18$ years, $N(\%)$ & $16(10.3)$ & $1(0.7)$ & $0(0.0)$ & $1(3.0)$ \\
\hline Male, $N(\%)$ & $90(58.1)$ & $83(56.1)$ & $25(65.8)$ & $19(57.6)$ \\
\hline Body mass index $\left(\mathrm{kg} / \mathrm{m}^{2}\right)^{* \mathrm{~b}}$ & $24.1 \pm 4.3$ & $23.8 \pm 3.5$ & $23.5 \pm 3.8$ & $23.1 \pm 3.5$ \\
\hline Systolic blood pressure $(\mathrm{mmHg})^{* b}$ & $121 \pm 17$ & $131 \pm 20$ & $135 \pm 18$ & $136 \pm 14$ \\
\hline Diastolic blood pressure $(\mathrm{mmHg})^{* \mathrm{~b}}$ & $73 \pm 12$ & $77 \pm 13$ & $79 \pm 13$ & $77 \pm 11$ \\
\hline Urinary protein $(\mathrm{g} /$ day $)(\text { or } \mathrm{g} / \mathrm{gCr})^{* \mathrm{bc}}$ & $6.8(4.8,10.4)$ & $4.4(2.9,6.3)$ & $7.5(4.5,10.7)$ & $5.1(3.4,6.9)$ \\
\hline$\geq 3.5 \mathrm{~g} /$ day (or $\mathrm{g} / \mathrm{gCr}), N(\%)$ & $133(86.4)$ & $101(68.7)$ & $35(92.1)$ & $24(72.7)$ \\
\hline $1.0-3.4$ & $19(12.3)$ & $43(29.3)$ & $3(7.9)$ & $9(27.3)$ \\
\hline $0.3-0.9$ & $0(0.0)$ & $3(2.0)$ & $0(0.0)$ & $0(0.0)$ \\
\hline$<0.3$ & $2(1.3)$ & $0(0.0)$ & $0(0.0)$ & $0(0.0)$ \\
\hline Serum creatinine $(\mathrm{mg} / \mathrm{dL})^{*}$ & $0.87(0.70,12.0)$ & $0.87(0.87,1.56)$ & $1.11(0.87,1.56)$ & $1.04(0.82,1.50)$ \\
\hline $\mathrm{eGFR}, \mathrm{mL} / \mathrm{min} / 1.73 \mathrm{~m}^{2 * \mathrm{~b}}$ & $68 \pm 27$ & $61 \pm 21$ & $52 \pm 22$ & $52 \pm 25$ \\
\hline$\geq 90 \mathrm{~mL} / \mathrm{min} / 1.73 \mathrm{~m}^{2}, N(\%)$ & $23(16.5)$ & $12(8.2)$ & $1(2.6)$ & $3(9.4)$ \\
\hline $60-89$ & $61(43.9)$ & $69(46.9)$ & $13(34.2)$ & $9(28.1)$ \\
\hline $45-59$ & $28(20.1)$ & $38(25.9)$ & $11(28.9)$ & $7(21.9)$ \\
\hline $30-44$ & $16(11.5)$ & $15(10.2)$ & $4(10.5)$ & $5(15.6)$ \\
\hline $15-29$ & $7(5.0)$ & $10(6.8)$ & $7(18.4)$ & $7(21.9)$ \\
\hline$<15$ & $4(2.9)$ & $3(2.0)$ & $2(5.3)$ & $1(3.1)$ \\
\hline Serum albumin $(\mathrm{g} / \mathrm{dL})^{*}$ & $1.7 \pm 0.6$ & $2.2 \pm 0.6$ & $1.9 \pm 0.7$ & $2.4 \pm 0.5$ \\
\hline Serum total cholesterol $(\mathrm{mg} / \mathrm{dL})^{* \mathrm{~b}}$ & $409 \pm 120$ & $320 \pm 95$ & $366 \pm 124$ & $290 \pm 89$ \\
\hline Hemoglobin A1c (\%) & $5.3 \pm 0.7$ & $5.5 \pm 0.9$ & $5.4 \pm 0.9$ & $5.2 \pm 0.5$ \\
\hline Use of RAS blockers, $N(\%)^{*}$ & $21(13.5)$ & $68(45.9)$ & $16(42.1)$ & $15(45.5)$ \\
\hline Use of statins, $N(\%)^{*}$ & $41(27.1)$ & $71(48.0)$ & $21(55.3)$ & $5(15.2)$ \\
\hline Use of antidiabetic drugs, $N(\%)^{*}$ & $7(4.5)$ & $4(2.7)$ & $2(5.3)$ & $3(9.1)$ \\
\hline Immunosuppressive therapy, $N(\%)^{*}$ & $153(98.7)$ & $127(85.8)$ & $35(92.1)$ & $24(72.7)$ \\
\hline $\begin{array}{l}\text { Time from kidney biopsy to immunosuppressive } \\
\text { therapy (day) }\end{array}$ & $3(-4,7)$ & $10(4,24)$ & $6(0,14)$ & $10(4,14)$ \\
\hline$<0$ day, $\mathrm{N}(\%)$ & $45(29.0)$ & $14(9.5)$ & $9(23.7)$ & $4(12.1)$ \\
\hline \multicolumn{5}{|c|}{ Immunosuppressive drugs within 24 months of immunosuppressive therapy, $N(\%)^{\mathrm{b}}$} \\
\hline Prednisolone & $150(98.7)$ & $118(95.2)$ & $35(100.0)$ & $22(100.0)$ \\
\hline Intravenous methylprednisolone & 48 (31.6) & $26(21.0)$ & $11(31.4)$ & $11(50.0)$ \\
\hline Cyclosporine & $54(35.5)$ & $70(56.5)$ & $22(62.9)$ & $6(27.3)$ \\
\hline Tacrolimus & $2(1.3)$ & $5(4.0)$ & $0(0.0)$ & $0(0.0)$ \\
\hline Cyclophosphamide & $1(0.7)$ & $9(4.8)$ & $0(0.0)$ & $3(13.6)$ \\
\hline Mizoribine & $8(5.3)$ & $23(18.5)$ & $0(0.0)$ & $1(4.5)$ \\
\hline Mycophenolate mofetil & $1(0.7)$ & $2(1.6)$ & $0(0.0)$ & $0(0.0)$ \\
\hline Rituximab & $4(2.6)$ & $1(0.8)$ & $1(2.9)$ & $0(0.0)$ \\
\hline
\end{tabular}

Mean \pm standard deviation; median $(25 \%, 75)$

$C r$ creatinine, $e G F R$ estimated glomerular filtration rate, FSGS focal segmental glomerulosclerosis, $M C D$ minimal change disease, $M N$ membranous nephropathy, $R A S$ renin-angiotensin system

${ }^{*} P<0.05$ for chi-square test, Fisher's exact test, ANOVA, or Kruskal-Wallis test, as appropriate

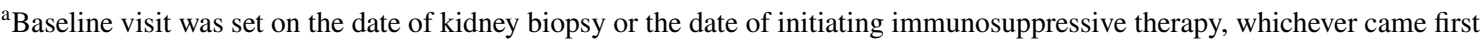

${ }^{b}$ Number of missing value: body mass index, $N=5$ in MCD; systolic and diastolic blood pressure, $N=4$ in MCD; Urinary protein, $N=1$ and 1 in MCD and FSGS; eGFR, $N=16,1$, and 1 in MCD, MN, and others because of $<18$ year of age; serum total cholesterol, $N=10,7$, and 3 in MCD, MN, and others; hemoglobin A1c, $N=45,31,8$, and 14 in MCD, MN, FSGS, and others; initial drugs within 1 month of immunosuppressive therapy, $N=1,3$, and 2 in $\mathrm{MCD}, \mathrm{MN}$, and others

${ }^{\mathrm{c}}$ Urinary protein/creatinine ratio (g/gCr) was used in $36(23.4 \%), 21(14.3 \%), 8(21.1 \%)$, and $6(18.2 \%)$ patients with MCD, MN, FSGS, and others, respectively, who had missing value of urinary protein (g/day) 
A Incomplete remission type 2 of proteinuria

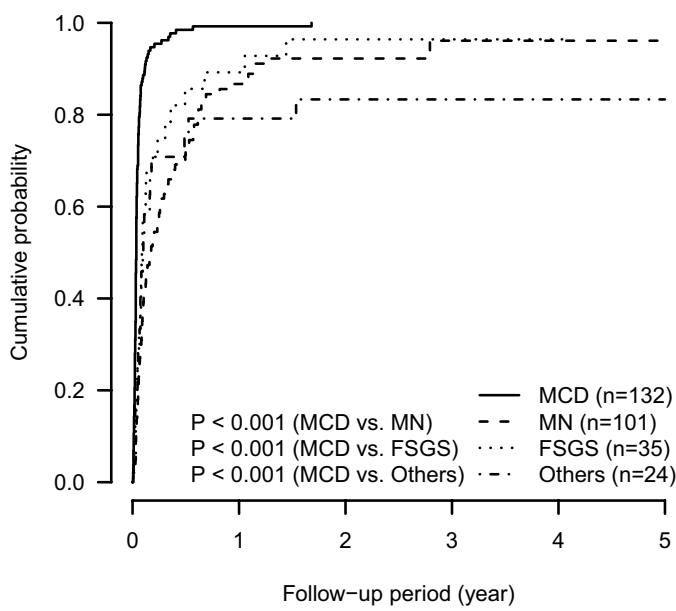

\section{Complete remission of proteinuria}

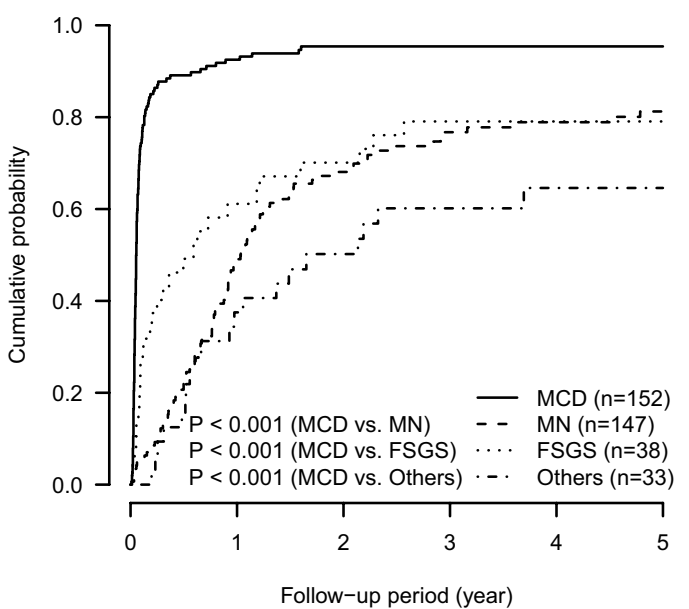

E $50 \%$ increase of serum creatinine or ESKD

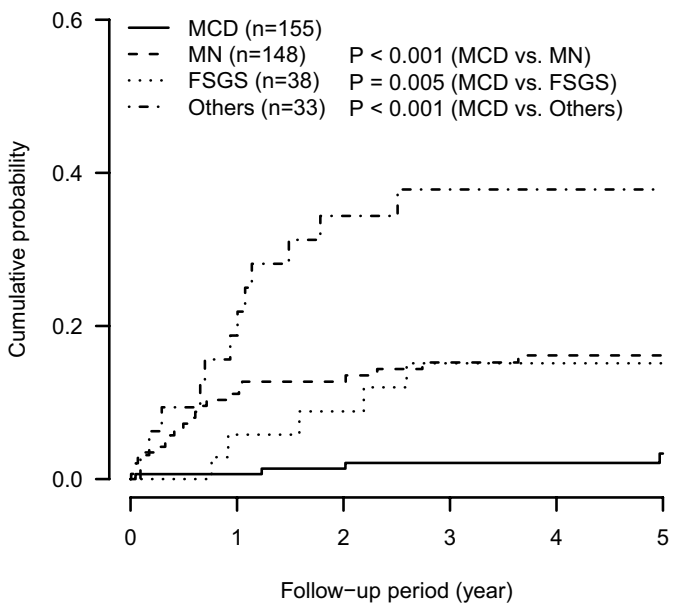

Fig. 2 Cumulative probabilities of major clinical outcomes: incomplete remission type 1 (a) and 2 (b), complete remission (c), relapse of proteinuria after complete remission (d), 50\% and $100 \%$ increase

\section{B Incomplete remission type 1 of proteinuria}

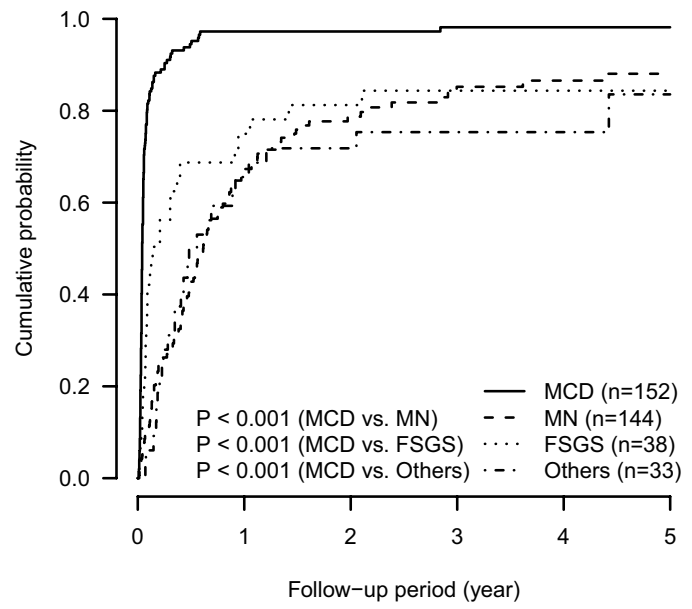

D Relapse of proteinuria after complete remissior

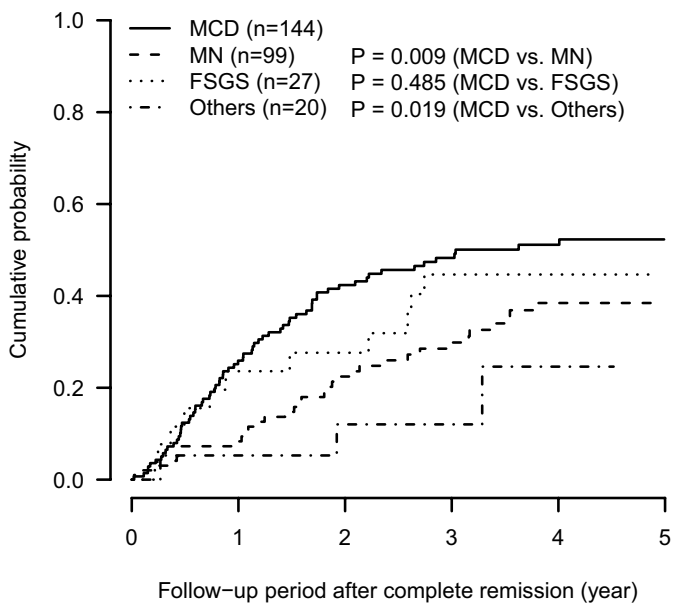

F $100 \%$ increase of serum creatinine or ESKD

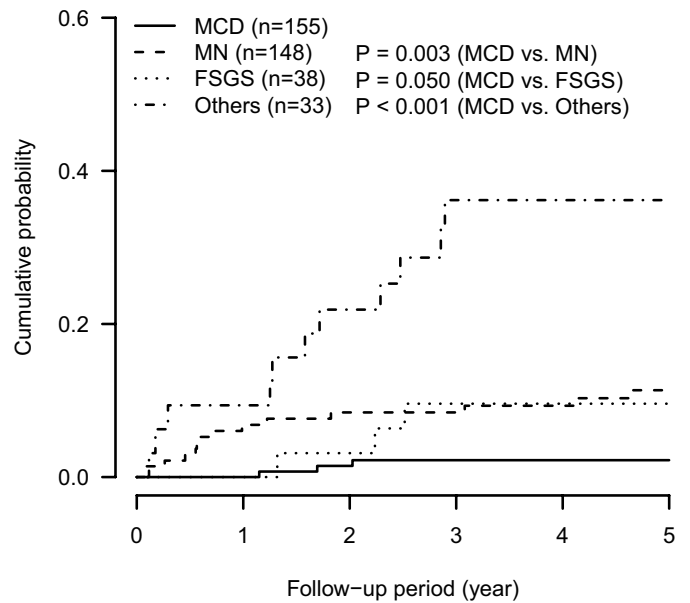

in serum creatinine and/or end-stage kidney disease (ESKD) (e, f), $\operatorname{ESKD}(\mathbf{g})$, all-cause mortality (h), and use of diabetic drugs (i) 
G End-stage kidney disease (ESKD)

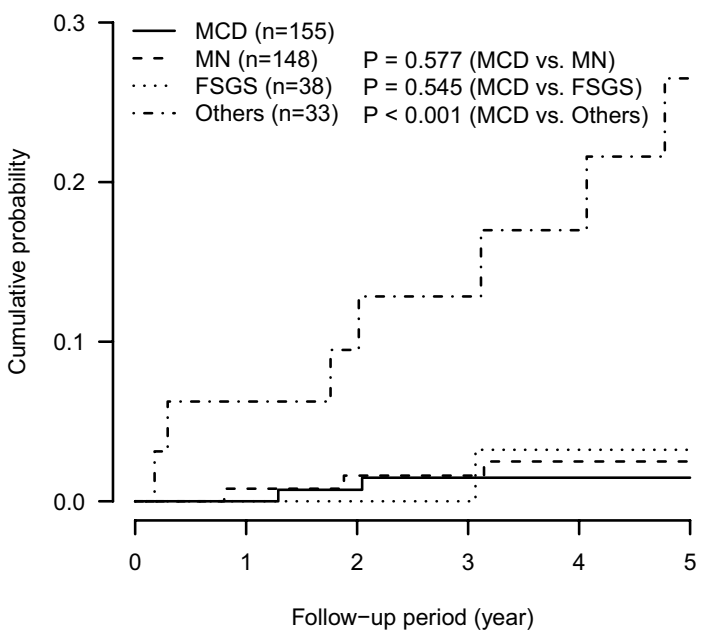

I Use of antidiabetic drugs

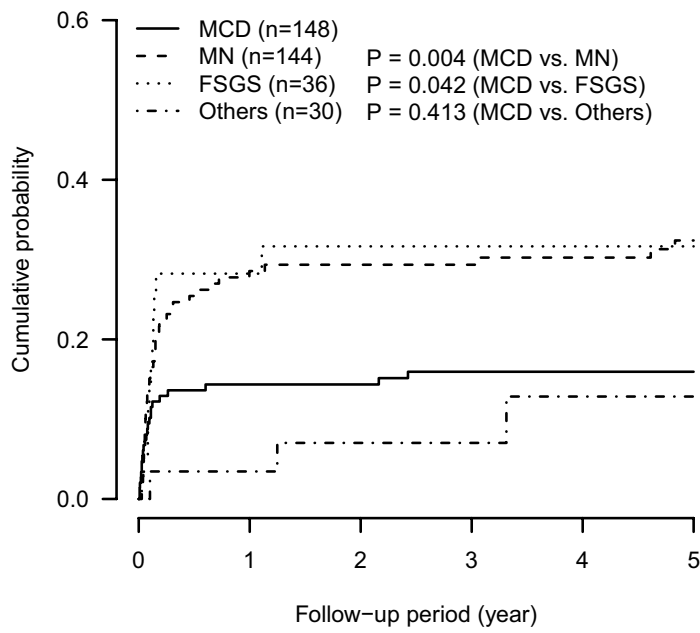

Fig. 2 (continued)

(cumulative probability of incomplete remission type 2 of proteinuria: 0.99 [95\% confidence interval $0.95,1.00$ ], 0.87 [0.78, 0.92], 0.89 [0.69, 0.94], and 0.79 [0.55, 0.90] in patients with MCD, MN, FSGS, and others, respectively) (Table 2). Complete remission of proteinuria (24-h urinary protein of $<0.3 \mathrm{~g} /$ day or urinary protein-to-creatinine ratio of $<0.3 \mathrm{~g} / \mathrm{gCr}$ ) was observed in approximately half of the patients with MN and FSGS within one year of the baseline visit. Their cumulative probabilities of complete remission increased by $75 \%$ within 3 years of the baseline visit $(0.77$ $[0.68,0.83]$ and $0.79[0.60,0.89]$ in patients with $\mathrm{MN}$ and FSGS, respectively), whereas only $60 \%$ in patients with other glomerulonephritides (0.60 [0.39, 0.74]) (Table 2). Patients with MCD, who had the highest cumulative probability of complete remission, also had the highest cumulative
H All-cause mortality

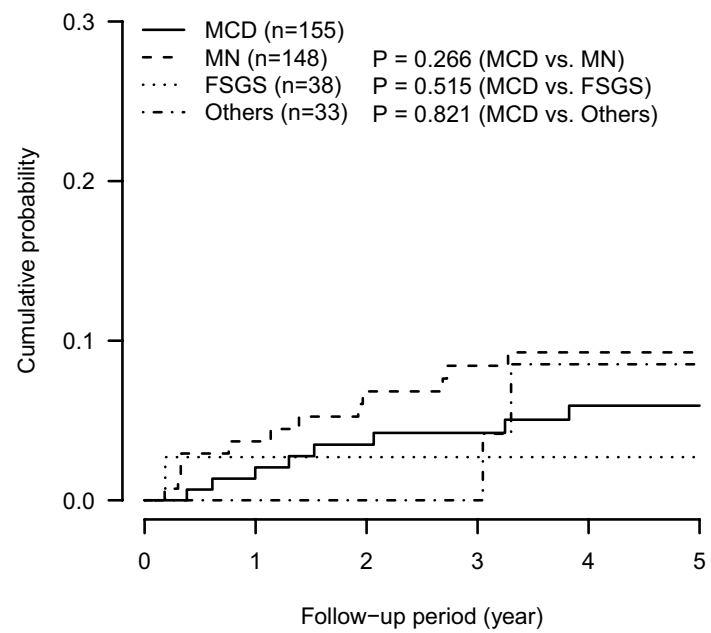

probability of relapse of proteinuria (Table 3). Approximately half of the patients with MCD experienced relapse of proteinuria within 3 years of the baseline visit (0.48 [0.39, $0.56])$. Relapse of proteinuria was also common in patients with FSGS $(0.44[0.20,0.60])$. Most patients with MCD and FSGS developed their first relapse during immunosuppressive therapy (79.1\% and $90.9 \%$, respectively).

Patients with other glomerulonephritides had a higher risk of decrease in GFR, followed by that in patients with MN, FSGS, and MCD (Figs. 2e-g, 3). The incidence rate per 1000 person-years of $50 \%$ increase in serum creatinine or ESKD was 6.5 (95\% confidence interval, 1.8, 16.7), 41.3 $(25.6,63.1), 34.6(11.2,80.8)$, and $121.5(62.8,212.2)$ in patients with MCD, MN, FSGS, and others, respectively (Fig. 3). Because of a short follow-up period of 5 years, the 
Table 2 Incidence of remission of proteinuria in primary nephrotic syndrome

\begin{tabular}{|c|c|c|c|c|}
\hline & MCD & $\mathrm{MN}$ & FSGS & Others \\
\hline \multicolumn{5}{|c|}{ Incomplete remission type 2 of proteinuria (urinary protein $<3.5 \mathrm{~g} / \mathrm{day}$ or $\mathrm{g} / \mathrm{gCr}$ ) } \\
\hline Baseline urinary protein $\geq 3.5 \mathrm{~g} /$ day (or $\mathrm{g} / \mathrm{Cr}$ ), $N$ & 132 & 101 & 35 & 24 \\
\hline Incidence of remission, $N(\%)$ & $132(100.0)$ & $90(89.1)$ & $32(91.6)$ & $20(83.3)$ \\
\hline Time to remission (day) & $13(8,20)$ & $50(21,146)$ & $30(17,54)$ & $28(17,59)$ \\
\hline \multicolumn{5}{|l|}{ Cumulative probability of remission $(95 \% \mathrm{CI})$} \\
\hline 1 month & $0.86(0.79,0.91)$ & $0.31(0.21,0.40)$ & $0.46(0.26,0.60)$ & $0.46(0.22,0.63)$ \\
\hline 2 months & $0.94(0.88,0.97)$ & $0.48(0.37,0.57)$ & $0.69(0.49,0.81)$ & $0.67(0.41,0.81)$ \\
\hline 1 year & $0.99(0.95,1.00)$ & $0.87(0.78,0.92)$ & $0.89(0.69,0.96)$ & $0.79(0.55,0.90)$ \\
\hline 3 years & NA & $0.96(0.82,0.99)$ & $0.96(0.76,0.99)$ & $0.93(0.59,0.93)$ \\
\hline \multicolumn{5}{|c|}{ Incomplete remission type 1 of proteinuria (urinary protein $<1.0 \mathrm{~g} /$ day or $\mathrm{g} / \mathrm{gCr}$ ) } \\
\hline Baseline urinary protein $\geq 1.0 \mathrm{~g} / \mathrm{day}$ ( or $\mathrm{g} / \mathrm{Cr}$ ), $N$ & 152 & 144 & 38 & 33 \\
\hline Incidence of remission, $N(\%)$ & $148(97.4)$ & $108(75.0)$ & $30(78.9)$ & $25(75.8)$ \\
\hline Time to remission (day) & $15(11,25)$ & $178(56,316)$ & $33(23,111)$ & $148(75,252)$ \\
\hline \multicolumn{5}{|l|}{ Cumulative probability of remission $(95 \% \mathrm{CI})$} \\
\hline 1 month & $0.77(0.69,0.83)$ & $0.09(0.04,0.14)$ & $0.32(0.15,0.45)$ & $0.06(0.00,0.14)$ \\
\hline 2 months & $0.88(0.82,0.92)$ & $0.20(0.13,0.27)$ & $0.50(0.31,0.64)$ & $0.09(0.00,0.19)$ \\
\hline 1 year & $0.97(0.93,0.99)$ & $0.67(0.58,0.75)$ & $0.75(0.55,0.86)$ & $0.66(0.44,0.79)$ \\
\hline 3 years & $0.98(0.94,0.99)$ & $0.85(0.77,0.91)$ & $0.84(0.64,0.93)$ & $0.75(0.54,0.87)$ \\
\hline \multicolumn{5}{|c|}{ Complete remission of proteinuria (urinary protein $<0.3 \mathrm{~g} /$ day or $\mathrm{g} / \mathrm{gCr}$ ) } \\
\hline Baseline urinary protein $\geq 0.3 \mathrm{~g} / \mathrm{day}$ (or $\mathrm{g} / \mathrm{Cr}$ ), $N$ & 152 & 147 & 38 & 33 \\
\hline Incidence of remission, $N(\%)$ & $144(94.7)$ & $100(68.0)$ & $28(73.7)$ & $20(60.6)$ \\
\hline Time to remission (day) & $19(13,31)$ & $292(152,443)$ & $82(31,283)$ & $290(185,558)$ \\
\hline \multicolumn{5}{|l|}{ Cumulative probability of remission $(95 \% \mathrm{CI})$} \\
\hline 1 month & $0.70(0.62,0.77)$ & $0.05(0.01,0.08)$ & $0.18(0.05,0.30)$ & $0.00(0.00,0.00)$ \\
\hline 2 months & $0.83(0.76,0.88)$ & $0.07(0.03,0.11)$ & $0.32(0.15,0.45)$ & $0.00(0.00,0.00)$ \\
\hline 1 year & $0.93(0.87,0.96)$ & $0.48(0.39,0.56)$ & $0.61(0.41,0.74)$ & $0.37(0.18,0.52)$ \\
\hline 3 years & $0.95(0.90,0.98)$ & $0.77(0.68,0.83)$ & $0.79(0.60,0.89)$ & $0.60(0.39,0.74)$ \\
\hline
\end{tabular}

Median $(25 \%, 75 \%)$

$C I$ confidence interval, $C r$ creatinine, $F S G S$ focal segmental glomerulosclerosis, $M C D$ minimal change disease, $M N$ membranous nephropathy, NA not assessed

incidence of ESKD was low in patients with MCD, MN, and FSGS (per 1000 person-years; 3.2 [0.4, 11.7], 5.3 [1.1, 15.4], 6.4 [0.2, 35.9], 59.1 [23.8, 121.8] in MCD, MN, FSGS, and others, respectively).

Compared with ESKD, all-cause mortality was more common in patients with MCD and MN (per 1000 personyears; $13.0[5.6,25.6]$ and $21.0[10.9,36.8]$ in MCD and $\mathrm{MN}$, respectively) (Figs. 2g-h, 3). The leading cause of death was infection $(N=11[47.8 \%])$, followed by malignancy $(N=7[30.4 \%])$, CVD $(N=2[8.7 \%])$ and others $(N=3[13.0 \%])$ (Fig. 3). In patients with MCD and MN, infection was the leading cause of death $(N=6[66.7 \%]$ and 5 [41.7\%] in MCD and MN, respectively). Although the incidence of hospitalization for infection was comparable among the four groups of glomerulonephritides (per 1000 person-years; 22.1 [11.8, 37.8], 16.2 [7.4, 30.8], 20.4 [4.2, 59.6], 25.1 [5.2, 73.3] in MCD, MN, FSGS, and others, respectively) (Fig. 3), patients with MCD were associated with hospitalization for infection at a marginally significant level than those with MN, after controlling for age and sex (vs. MN; MCD, adjusted hazard ratio 2.41 [95\% confidence interval 0.98, 5.94], $P=0.06$; FSGS, 1.58 [0.43, 5.88], $P=0.5$; others, $1.78(0.48,6.58), P=0.4)$ (Table 4). After an additional adjustment for serum creatinine, MCD was significantly associated with hospitalization for infection (Model 2: MCD, 2.44 [1.00, 5.95], $P=0.05$; FSGS, 1.48 $[0.40,5.50], P=0.6$; other glomerulonephritides, 1.26 [0.30, 5.29], $P=0.6$ ). A further adjustment for urinary protein confirmed their associations (Model 3).

The use of antidiabetic drugs was common especially among patients with MN and FSGS (Fig. 2I). Their cumulative incidence of use of antidiabetic drugs was approximately $30 \%$ one year after the baseline visit (1-year cumulative probability in MCD, MN, FSGS, and other glomerulonephritides: 0.14 [0.08, 0.20], 0.28 [0.20, 0.35], 0.28 $[0.12,0.42]$, and $0.03[0.00,0.10]$, respectively). During the 
Table 3 Incidence of relapse of proteinuria after complete remission in primary nephrotic syndrome

\begin{tabular}{|c|c|c|c|c|}
\hline & $\mathrm{MCD}$ & $\mathrm{MN}$ & FSGS & Others \\
\hline Follow-up after complete remission $>0$ day, $N$ & 144 & 99 & 27 & 20 \\
\hline Incidence of relapse, $N(\%)$ & $67(46.5)$ & $33(33.3)$ & $11(40.7)$ & $3(15.0)$ \\
\hline Time from complete remission to 1 st relapse (year) & $0.96(0.50,1.69)$ & $1.59(1.03,2.59)$ & $0.88(0.43,2.40)$ & $1.92(1.10,2.60)$ \\
\hline \multicolumn{5}{|l|}{ Use of immunosuppressive drugs at 1 st relapse, $N(\%)$} \\
\hline Prednisolone & $47(70.1)$ & $15(45.5)$ & $9(81.8)$ & $2(66.7)$ \\
\hline Cyclosporine & $9(13.4)$ & $8(24.2)$ & $6(54.5)$ & $1(33.3)$ \\
\hline Tacrolimus & $0(0.0)$ & $0(0.0)$ & $0(0.0)$ & $0(0.0)$ \\
\hline Cyclophosphamide & $1(1.5)$ & $1(3.0)$ & $0(0.0)$ & $0(0.0)$ \\
\hline Mizoribine & $2(3.0)$ & $1(3.0)$ & $0(0.0)$ & $0(0.0)$ \\
\hline Mycophenolate mofetil & $0(0.0)$ & $0(0.0)$ & $0(0.0)$ & $0(0.0)$ \\
\hline Any drugs & $53(79.1)$ & $17(51.5)$ & $10(90.9)$ & $2(66.7)$ \\
\hline \multicolumn{5}{|l|}{ Cumulative probability of relapse $(95 \% \mathrm{CI})$} \\
\hline 1 year & $0.26(0.18,0.33)$ & $0.08(0.03,0.14)$ & $0.24(0.05,0.38)$ & $0.05(0.00,0.15)$ \\
\hline 2 years & $0.42(0.33,0.50)$ & $0.22(0.13,0.30)$ & $0.28(0.08,0.43)$ & $0.12(0.00,0.26)$ \\
\hline 3 years & $0.48(0.39,0.56)$ & $0.30(0.20,0.39)$ & $0.44(0.20,0.60)$ & $0.12(0.00,0.26)$ \\
\hline
\end{tabular}

Median $(25 \%, 75 \%)$

$C I$ confidence interval, $C r$ creatinine, $F S G S$ focal segmental glomerulosclerosis, $M C D$ minimal change disease, $M N$ membranous nephropathy

5-year follow-up period, other clinical outcomes were relatively rare, including hospitalization for malignancy, CVD, thrombosis, aseptic osteonecrosis, and peptic ulcer (Fig. 3).

\section{Discussion}

The present 5-year cohort study, which included 374 patients with primary nephrotic syndrome in 55 hospitals in Japan, clarified the incidence rate of major clinical outcomes and disclosed that the incidence of all-cause mortality was higher than that of ESKD in patients with two major glomerulonephritides, MCD and MN. Hospitalization for infection, the leading cause of all-cause mortality, was significantly more common in patients with MCD than those with MN, suggesting that patients with MCD were vulnerable to infection. Several advantages of the present study include the nature of the cohort study design, the inclusion of two major glomerulonephritides, MCD and MN, the measurements of a wide variety of major clinical outcomes, and the collection of recent real-world clinical data in the most recent decade between 2009 and 2015.

Few studies have compared the incidence rates of ESKD and all-cause mortality among patients with primary nephrotic syndrome. A Korean single-center retrospective cohort study, including 187, 232, and 251 patients with MCD, MN, and FSGS, showed that all-cause mortality was more common than ESKD in MCD and MN during the median observational period of 7.5 years, whereas ESKD was more common than all-cause mortality in FSGS [21]. A similar finding was also reported in a Taiwanese single-center retrospective cohort study with a median observational period of 5.9 years, including 109, 209, and 132 patients with MCD, MN, and FSGS, respectively [22]. However, these studies did not clarify the causes of mortality. After confirming that all-cause mortality was more common than ESKD in patients with MCD and MN, the present multicenter cohort study identified infection as the leading cause of mortality (Fig. 3) and disclosed that patients with MCD were more vulnerable to infection compared to patients with MN (Table 4). One of the plausible reasons for the higher incidence rate of infection in patients with $\mathrm{MCD}$ might be due to the higher incidence of relapses of proteinuria with add-on use of immunosuppressive drugs. Compared to patients with MN, patients with MCD had a higher risk of relapse of proteinuria during immunosuppressive therapy (Table 3), probably leading to the higher doses of immunosuppressive drugs. Unfortunately, the dose of each immunosuppressive drug during the immunosuppressive therapy was not available in the present study. Further research with details of immunosuppressive drugs are essential to assess an association between immunosuppressive therapy and infection.

Comparable with the results of the present cohort study, previous retrospective cohort studies on MCD reported that infection was one of the most common adverse events [23-27] and one of the leading causes of mortality [28]. To suppress the incidence of infection, a lower dose of and/or shorter term immunosuppressive therapy is desirable. Among pediatric patients with corticosteroid-sensitive nephrotic syndrome, two randomized trials in Japan [29] and India [30] recently demonstrated that the incidence 
Fig. 3 Incidence rates of major outcomes in primary nephrotic syndrome; causes of mortality included infection $(N=6,5$, and 1 in MCD, MN, and FSGS, respectively), malignancy $(N=1,5$, and 1 in $\mathrm{MCD}, \mathrm{MN}$, and other glomerulonephritides, respectively), cardiovascular disease ( $N=1$ and 1 in MCD and MN, respectively) and others $(N=1,1$, and 1 in $\mathrm{MCD}, \mathrm{MN}$, and other glomerulonephritides, respectively)

of relapse of proteinuria was comparable between conventional 6-month corticosteroid therapy and 3-month corticosteroid therapy. In adult patients with MCD, only low-quality evidences in this regard are available. Several guidelines suggested longer corticosteroid therapy; the Kidney Disease Improving Global Outcomes (KDIGO) clinical guideline for glomerulonephritis suggested a daily dose of $1.0 \mathrm{mg} / \mathrm{kg}$ of prednisolone or an alternate-day single dose of $2 \mathrm{mg} / \mathrm{kg}$ tapered slowly over a total period of up to 6 months [12] and the Japanese evidence-based clinical practice guideline for nephrotic syndrome suggest $0.6-0.8 \mathrm{mg} / \mathrm{kg}$ of prednisolone tapered within 2 years [13]. Compared with these conventional long corticosteroid therapies, an intriguing Japanese observational study of adult patients with MCD suggested clinical advantages of a 2-month corticosteroid therapy, the lower incidence of adverse events, including diabetes and infection [31]. Because nephrologists might possibly maintain adult patients with MCD on corticosteroids for very long [32], an optimal immunosuppressive therapy should be explored in well-designed clinical studies to prevent critical events associated with immunosuppressive therapy, including infection.

The present study has several limitations. First, the incidence of all-cause mortality $(N=22)$ and $\operatorname{ESKD}(N=12)$ was small; thus, the findings of the present study might not be reproducible. The JNSCS is planning to extend the 5-year follow-up period to 10 years, providing more precise details regarding the clinical impacts of major outcomes in patients with primary nephrotic syndrome. Second, the small number of patents with FSGS and other glomerulonephritides hindered statistically meaningful analyses in JNSCS. The results of the present study suggested the cumulative probabilities of complete remission of proteinuria and an increase of serum creatinine of FSGS were comparable to those of MN (Fig. 2c, e, f), although the higher rate of relapse of proteinuria of FSGS, which was comparable to that of MCD (Fig. 2d). Because the higher probability of relapse might contribute to the higher risk of infection in patients with MCD, those with FSGS might be similarly vulnerable to infection. To clarify their clinical courses, a larger cohort study is needed. Third, the incidences of some outcomes were dependent on the practice patterns of each hospital. For example, the thresholds of plasma glucose concentration and/or hemoglobin A1c to start antidiabetic drugs might be different among the hospitals.

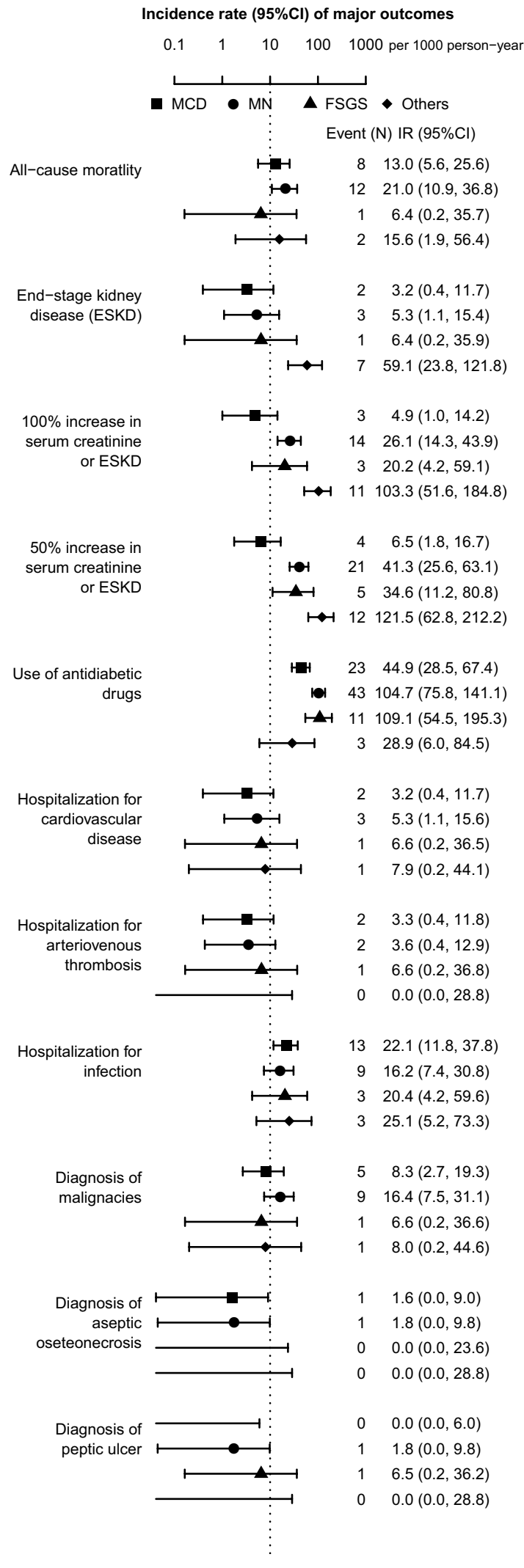


Table 4 Incidence of hospitalization for infection in primary nephrotic syndrome

\begin{tabular}{lllll}
\hline & MCD & MN & FSGS & Others \\
\hline Incidence of infection, $N(\%)$ & $13(8.4)$ & $9(6.1)$ & $3(7.9)$ & $3(9.1)$ \\
Follow-up period (year) & $4.9(2.8,5.0)$ & $5.0(2.7,5.0)$ & $5.0(3.9,5.0)$ & $4.1(2.6,5.0)$ \\
$\begin{array}{l}\text { Incidence rate of infection, per } \\
\text { 1000 person-years }\end{array}$ & $22.1(11.8,37.8)$ & $16.2(7.4,30.8)$ & $20.4(4.2,59.6)$ & $25.1(5.2,73.3)$ \\
Hazard ratio (95\% CI) & & & & \\
Unadjusted model & $1.33(0.57,3.11)$ & 1.00 (reference) & $1.27(0.35,4.71)$ & $1.48(0.40,5.46)$ \\
Multivariable-adjusted model 1 & $2.41(0.98,5.94)^{\dagger}$ & 1.00 (reference) & $1.58(0.43,5.88)$ & $1.78(0.48,6.58)$ \\
Multivariable-adjusted model 2 & $2.44(1.00,5.95)^{\ddagger}$ & 1.00 (reference) & $1.48(0.40,5.50)$ & $1.26(0.30,5.29)$ \\
Multivariable-adjusted model 3 & $2.56(1.04,6.34)^{\ddagger}$ & 1.00 (reference) & $1.55(0.41,5.83)$ & $1.22(0.29,5.19)$ \\
\hline
\end{tabular}

$C I$ confidence interval, FSGS focal segmental glomerulosclerosis, $M C D$ minimal change disease, $M N$ membranous nephropathy

${ }^{\dagger} P=0.06$

${ }^{\ddagger} P<0.05$

${ }^{\text {a }}$ Model 1 adjusted for baseline age and stratified by sex; Model 2 adjusted for baseline age and serum creatinine and stratified by sex; Model 3 adjusted for baseline age, serum creatinine, and urinary protein and stratified by sex
In conclusion, the JNSCS revealed that patients with MCD and MN had a higher risk of all-cause mortality than that of ESKD. Patients with MCD were more vulnerable to infection, the leading cause of mortality, compared to patients with MN. These results provide pivotal information that identifies the treatment goals of primary nephrotic syndrome with the recent immunosuppressive therapy. Nephrologists might possibly pay more attention to infection in patients with primary nephrotic syndrome.

Acknowledgements JNSCS was supported by a Grant-in-Aid for intractable Renal Diseases Research, Research on rare and intractable diseases, Health and Labour Sciences Research Grants for the Ministry of Health, Labor, and Welfare of Japan. JNSCS has been supported by a large number of investigator in 56 participating facilities; Hokkaido University Hospital, Sapporo, Hokkaido (Saori Nishio, Yasunobu Ishikawa, Daigo Nakazawa, and Tasuku Nakagaki); JCHO Sendai Hospital, Sendai, Miyagi (Toshinobu Sato, Mitsuhiro Sato, and Satoru Sanada); Tohoku University Hospital, Sendai, Miyagai (Hiroshi Sato, Mariko Miyazaki, Takashi Nakamichi, Tae Yamamoto, Kaori Narumi, and Gen Yamada); Yamagata University Hospital, Yamagata, Yamagata (Tsuneo Konta, and Kazuobu Ichikawa); Fukushima Medical University Hospital, Fukushima, Fukushima (Junichiro James Kazama, Tsuyoshi Watanabe, Koichi Asahi, Yuki Kusano, and Kimio Watanabe); University of Tsukuba Hospital, Tsukuba, Ibaraki (Kunihiro Yamagata, Joichi Usui, Shuzo Kaneko, and Tetsuya Kawamura); Gunma University Hospital, Maeashi, Gunma (Keiju Hiromura, Akito Maeshima, Yoriaki Kaneko, Hidekazu Ikeuchi, Toru Sakairi, and Masao Nakasatomi); Saitama Medical Center, Saitama Medical University, Kawagoe, Saitama (Hajime Hasegawa, Takatsugu Iwashita, Taisuke Shimizu, Koichi Kanozawa, Tomonari Ogawa, Kaori Takayanagi, and Tetsuya Mitarai); Department of Nephrology, Saitama Medical University, Irumagun, Saitama (Hirokazu Okada, Tsutomu Inoue, Hiromichi Suzuki, and Kouji Tomori); Tokyo Women's Medical University, Shinjuku-ku, Tokyo (Kosaku Nitta, Takahito Moriyama, Akemi Ino, and Masayo Sato); Teikyo University School of Medicine, Itabashi-ku, Tokyo (Shunya Uchida, Hideaki Nakajima, Hitoshi Homma, Nichito Nagura, Yoshifuru Tamura, Shigeru Shibata, and Yoshihide Fujigaki); Juntendo Faculty of Medicine, Bunkyo-ku, Tokyo (Yusuke Suzuki, Yukihiko Takeda, Isao Osawa, and Teruo Hidaka); St. Marianna University, Kawasaki, Kanagawa (Yugo Shibagaki, Sayuri Shirai,
Daisuke Ichikawa, Tsutomu Sakurada, Tomo Suzuki, and Mikako Hisamichi); Niigata University Medical and Dental Hospital, Niigata, Niigata (Ichiei Narita, Naohumi Imai, Yumi Ito, Shin Goto, Yoshikatsu Kaneko, and Rhohei Kaseda); Kanazawa Medical University, Uchinada, Ishikawa (Hitoshi Yokoyama, Keiji Fujimoto, and Norifumi Hayashi); Kanazawa Univeristy Hospital, Kanazawa, Ishikawa (Takashi Wada, Miho Shimizu, Kengo Furuichi, Norihiko Sakai, Yasunori Iwata, Tadashi Toyama, and Shinji Kitajima); National Hospital Organization Kanazawa Medical Center, Kanazawa, Ishikawa (Kiyoki Kitagawa); Ogaki Municipal Hospital, Ogaki, Gifu (Hiroshi Sobajima, Norimi Ohashi, So Oshitani, and Kiyohito Kawashima); Gifu Prefectural Tajimi Hospital, Tajimi, Gifu (Tetsushi Mimura); Hamamatsu University Hospital, Hamamatsu, Shizuoka (Hidemo Yasuda, Akira Hishida, and Yoshihide Fujigaki); Shizuoka General Hospital, Shizuoka, Shizuoka (Satoshi Tanaka, and Noriko Mori); Chutoen General Medical Center, Kakegawa, Shizuoka (Toshiyuki Akahori, and Yutaka Fujita); Nagoya University Graduate School of Medicine, Nagoya, Aichi (Shoichi Maruyama, Naotake Tsuboi, Tomoki Kosugi, Takuji Ishimoto, Takayuki Katsuno, Noritoshi Kato, and Waichi Sato); Japanese Red Cross Nagoya Daini Hospital, Nagoya, Aichi (Asami Takeda, Kunio Morozumi, Yasuhiro Ohtsuka, Hibiki Shinjo, and Akihito Tanaka); Fujita Health University School of Medicine, Toyoake, Aichi (Hiroki Hayashi, Yukio Yuzawa, Midori Hasegawa, Daijo Inaguma, Shigehisa Koide, and Kazuo Takahashi); Konan Kosei Hospital, Konan, Aichi (Takeyuki Hiramatsu, Shinji Furuta, and Hideaki Ishikawa); Anjo Kosei hospital, Anjo, Aichi (Hirofumi Tamai, and Takatoshi Morinaga); Ichinomiya Municipal Hospital, Ichinomiya, Aichi (Arimasa Shirasaki, Toshiki Kimura, and Mina Kato); Japanese Red Cross Nagoya Daiichi Hospital, Nagaoya, Aichi (Shizunori Ichida, and Nobuhide Endo); Kasugai Municipal Hospital, Kasugai, Aichi (Tomohiko Naruse, Yuzo Watanabe, and Yosuke Saka); Kainan hospital, Yatomi, Aichi (Satashi Suzuki, Michiko Yamazaki, and Rieko Morita); Masuko Memorial Hospital, Nagoya, Aichi (Kunio Morozumi, Kunio Morozumi, Kaoru Yasuda, Chika Kondo, Takahiro Morohiro, Rho Sato, and Yuichi Shirasawa); Chubu Rosai Hospital, Nagoya, Aichi (Yoshiro Fujita, Hideaki Shimizu, and Tatsuhito Tomino); Handa City Hospital, Handa, Aichi (Makoto Mizutani); Yokkaichi Municipal Hospital, Yokkaichi, Mie (Yosuke Saka, Hiroshi Nagaya, and Makoto Yamaguchi); Kitano Hospital, Osaka, Osaka (Tatsuo Tsukamoto, Eri Muso, Hiroyuki Suzuki, Tomomi Endo, and Hiroko Kakita); Toyonaka Municipal Hospital, Toyonaka, Osaka (Megumu Fukunaga); Osaka General Medical Center, Osaka, Osaka (Tatsuya Shoji, and Terumasa Hayashi); Osaka City University Hospital, Osaka, Osaka (Eiji Ishimura, Akihiro Tsuda, 
Shinya Nakatani, Ikue Kobayashi, Mitsuru Ichii, Akinobu Ochi, and Yoshiteru Ohno); Osaka Univeristy hospital, Suita, Osaka (Yoshitaka Isaka, Enyu Imai, Yasuyuki Nagasawa, Hirotsugu Iwatani, Ryohei Yamamoto, and Tomoko Namba); Kobe University hospital, Kobe, Hyogo (Shunsuke Goto MD, and Shinichi Nishi); Nara Medical University Hospital, Kashihara, Nara (Yasuhiro Akai, Ken-ichi Samejima, Masaru Matsui, Miho Tagawa, Kaori Tanabe, and Hideo Tsushima); Wakayama Medical University Hospital, Wakayama, Wakayama (Takashi Shigematsu MD, Masaki Ohya, Shigeo Negi, and Toru Mima); Shimane University Hospital, Izumo, Shimane (Takafumi Ito); Okayama Univeristy Hospital, Okayama, Okayama (Hitoshi Sugiyama, Keiko Tanaka, Toshio Yamanari, Masashi Kitagawa, Akifumi Onishi, and Koki Mise); Kawasaki Medical School, Kurashiki, Okayama (Naoki Kashihara, Tamaki Sasaki, Sohachi Fujimoto, and Hajime Nagasu); Graduate School of Medicine, The University of Tokushima,Tokushima,Tokushima (Kojiro Nagai, and Toshio Doi); Kagawa University, Miki-cho, Takamatsu, Japan (Tadashi Sofue, Hideyasu Kiyomoto, Kumiko Moriwaki, Taiga Hara, Yoko Nishijima, Yoshio Kushida, and Tetsuo Minamino); Kochi Medical School, Kochi University, Nankoku, Kochi (Yoshio Terada, Taro Horino, Yoshinori Taniguchi, Kosuke Inoue, Yoshiko Shimamura, and Tatsuki Matsumoto); Kyushu University Hospital, Fukuoka, Fukuoka (Kazuhiko Tsuruya, Hisako Yoshida, Naoki Haruyama, Shunsuke Yamada, Akihiro Tsuchimoto, and Yuta Matsukuma); Fukuoka University Hospital, Fukuoka, Fukuoka (Kosuke Masutani, Yasuhiro Abe, Aki Hamauchi, Tetsuhiko Yasuno, and Kenji Ito); Kurume University Hospital, Kurume, Fukuoka (Kei Fukami, Junko Yano, Chika Yoshida, Yuka Kurokawa, and Nao Nakamura); National Fukuoka Higashi Medical Center, Koga, Fukuoka (Ritsuko Katafuchi, Hiroshi Nagae, Shumei Matsueda, and Kazuto Abe); Nagasaki University Hospital, Nagasaki, Nagasaki (Tomoya Nishino, Tadashi Uramatsu, and Yoko Obata); Miyazaki University Hospital, Miyazaki, Miyazaki (Shouichi Fujimoto, Yuji Sato, Masao Kikuchi, Ryuzo Nishizono, Takashi Iwakiri, and Hiroyuki Komatsu).

\section{Compliance with ethical standards}

Conflict of interest The authors have declared that no conflict of interest exists.

Ethical approval All procedures performed in JNSCS involving human participants were in accordance with the ethical standards of the institutional research committee at which the studies were conducted (IRB approval number 17035-4 at the ethics committee of Osaka University Hospital) and with the 1964 Helsinki declaration and its later amendments or comparable ethical standards.

Informed consent Informed consent was obtained from participants in 54 hospitals and a single hospital used an opt-out approach to informed consent, according to Japanese Ethical Guidelines for Medical and Health Research Involving Human Subjects.

Open Access This article is licensed under a Creative Commons Attribution 4.0 International License, which permits use, sharing, adaptation, distribution and reproduction in any medium or format, as long as you give appropriate credit to the original author(s) and the source, provide a link to the Creative Commons licence, and indicate if changes were made. The images or other third party material in this article are included in the article's Creative Commons licence, unless indicated otherwise in a credit line to the material. If material is not included in the article's Creative Commons licence and your intended use is not permitted by statutory regulation or exceeds the permitted use, you will need to obtain permission directly from the copyright holder. To view a copy of this licence, visit http://creativecommons.org/licenses/by/4.0/.

\section{References}

1. Agrawal S, Zaritsky JJ, Fornoni A, Smoyer WE. Dyslipidaemia in nephrotic syndrome: mechanisms and treatment. Nat Rev Nephrol. 2017;14:57-70.

2. Shiiki H, Saito T, Nishitani Y, Mitarai T, Yorioka N, Yoshimura A, et al. Prognosis and risk factors for idiopathic membranous nephropathy with nephrotic syndrome in Japan. Kidney Int. 2004;65:1400-7.

3. Troyanov S, Wall CA, Miller JA, Scholey JW, Cattran DC, Toronto Glomerulonephritis Registry Group. Focal and segmental glomerulosclerosis: definition and relevance of a partial remission. J Am Soc Nephrol. 2005;16:1061-8.

4. Kono M, Yasuda S, Kato M, Kanetsuka Y, Kurita T, Fujieda Y, et al. Long-term outcome in Japanese patients with lupus nephritis. Lupus. 2014;23:1124-32.

5. Christiansen CF, Schmidt M, Lamberg AL, Horváth-Puhó E, Baron JA, Jespersen B, et al. Kidney disease and risk of venous thromboembolism: a nationwide population-based case-control study. J Thromb Haemost. 2014;12:1449-544.

6. Wu HM, Tang JL, Cao L, Sha ZH, Li Y. Interventions for preventing infection in nephrotic syndrome. Cochrane Datab Syst Rev. 2012;4:CD003964.

7. Christiansen CF, Onega T, Sværke C, Körmendiné Farkas D, Jespersen B, Baron JA, et al. Risk and prognosis of cancer in patients with nephrotic syndrome. Am J Med. 2014;127(871-7):e1.

8. Ordoñez JD, Hiatt RA, Killebrew EJ, Fireman BH. The increased risk of coronary heart disease associated with nephrotic syndrome. Kidney Int. 1993;44:638-42.

9. Wakasugi M, Kazama JJ, Narita I. Premature mortality due to nephrotic syndrome and the trend in nephrotic syndrome mortality in Japan, 1995-2014. Clin Exp Nephrol. 2018;22:55-60.

10. Sugiyama H, Yokoyama H, Sato H, Saito T, Kohda Y, Nishi S, et al. Japan renal biopsy registry: the first nationwide, web-based, and prospective registry system of renal biopsies in Japan. Clin Exp Nephrol. 2011;15:493-503.

11. McGrogan A, Franssen CFM, De Vries CS. The incidence of primary glomerulonephritis worldwide: a systematic review of the literature. Nephrol Dial Transpl. 2011;26:414-30.

12. Kidney Disease Improving Global Outcomes. KDIGO clinical practice guideline for glomerulonephritis. Kidnet Int Suppl. 2012;2:1-274

13. Nishi S, Ubara Y, Utsunomiya Y, Okada K, Obata Y, Kai H, et al. Evidence-based clinical practice guidelines for nephrotic syndrome 2014. Clin Exp Nephrol. 2016;20:342-70.

14. Chen Y, Schieppati A, Chen X, Cai G, Zamora J, Giuliano GA, et al. Immunosuppressive treatment for idiopathic membranous nephropathy in adults with nephrotic syndrome. Cochrane database Syst Rev. 2014;10:CD004293.

15. Ren S, Wang Y, Xian L, Toyama T, Jardine M, Li G, et al. Comparative effectiveness and tolerance of immunosuppressive treatments for idiopathic membranous nephropathy: a network meta-analysis. PLoS ONE. 2017;12:1-15.

16. Van Spall HGC, Toren A, Kiss A, Fowler RA. Eligibility criteria of randomized controlled trials published in high-impact general medical journals: a systematic sampling review. JAMA. 2007;297:1233-40.

17. Yamamoto R, Imai E, Maruyama S, Yokoyama H, Sugiyama H, Nitta K, et al. Regional variations in immunosuppressive therapy in patients with primary nephrotic syndrome: the Japan nephrotic syndrome cohort study. Clin Exp Nephrol. 2018;22:1266-80.

18. Churg J, Bernstein J, Glassock R. Renal disease, classification and atlas of glomerular disease. 2nd ed. New York: Igaku-Shoin Medical Pub; 1995. 
19. D’Agati VD, Fogo AB, Bruijn JA, Jennette JC. Pathologic classification of focal segmental glomerulosclerosis: a working proposal. Am J Kidney Dis. 2004;43:368-82.

20. Matsuo S, Imai E, Horio M, Yasuda Y, Tomita K, Nitta K, et al. Revised equations for estimated GFR from serum creatinine in Japan. Am J Kidney Dis. 2009;53:982-92.

21. Lee H, Kim DK, Oh K-H, Joo KW, Kim YS, Chae D-W, et al. Mortality and renal outcome of primary glomerulonephritis in Korea: observation in 1943 biopsied cases. Am J Nephrol. 2013;37:74-83.

22. Chou YH, Lien YC, Hu FC, Lin WC, Kao CC, Lai CF, et al. Clinical outcomes and predictors for ESRD and mortality in primary GN. Clin J Am Soc Nephrol. 2012;7:1401-8.

23. Waldman M, Crew RJ, Valeri A, Busch J, Stokes B, Markowitz G, et al. Adult minimal-change disease: clinical characteristics, treatment, and outcomes. Clin J Am Soc Nephrol. 2007;2:445-53.

24. Shinzawa M, Yamamoto R, Nagasawa Y, Oseto S, Mori D, Tomida K, et al. Age and prediction of remission and relapse of proteinuria and corticosteroid-related adverse events in adult-onset minimal-change disease: a retrospective cohort study. Clin Exp Nephrol. 2013;17:839-47.

25. Maas RJ, Deegens JK, Beukhof JR, Reichert LJ, ten Dam MA, Beutler JJ, et al. The clinical course of minimal change nephrotic syndrome with onset in adulthood or late adolescence: a case series. Am J Kidney Dis. 2017;69:637-46.

26. Fenton A, Smith SW, Hewins P. Adult minimal-change disease: observational data from a UK centre on patient characteristics, therapies, and outcomes. BMC Nephrol. 2018;19:1-8.
27. Ozeki T, Ando M, Yamaguchi M, Katsuno T, Kato S, Yasuda $\mathrm{Y}$, et al. Treatment patterns and steroid dose for adult minimal change disease relapses: a retrospective cohort study. PLoS ONE. 2018;13:e0199228.

28. Szeto C-C, Lai FM-M, Chow K-M, Kwan BC-H, Kwong VW-K, Leung C-B, et al. Long-term outcome of biopsy-proven minimal change nephropathy in Chinese adults. Am J Kidney Dis. 2015;65:710-8.

29. Yoshikawa N, Nakanishi K, Sako M, Oba MS, Mori R, Ota E, et al. A multicenter randomized trial indicates initial prednisolone treatment for childhood nephrotic syndrome for two months is not inferior to six-month treatment. Kidney Int. 2015;87:225-32.

30. Sinha A, Saha A, Kumar M, Sharma S, Afzal K, Mehta A, et al. Extending initial prednisolone treatment in a randomized control trial from 3 to 6 months did not significantly influence the course of illness in children with steroid-sensitive nephrotic syndrome. Kidney Int. 2015;87:217-24.

31. Ozeki T, Katsuno T, Hayashi H, Kato S, Yasuda Y, Ando M, et al. Short-term steroid regimen for adult steroid-sensitive minimal change disease. Am J Nephrol. 2019;49:54-63.

32. Bomback AS. Shortening the duration of corticosteroid exposure in minimal change disease: can we treat adults like children? Am J Nephrol. 2019;49:52-3.

Publisher's Note Springer Nature remains neutral with regard to jurisdictional claims in published maps and institutional affiliations.

\section{Affiliations}

Ryohei Yamamoto ${ }^{1,2}$ (1) $\cdot$ Enyu Imai ${ }^{3} \cdot$ Shoichi Maruyama $^{4} \cdot$ Hitoshi Yokoyama $^{5} \cdot$ Hitoshi Sugiyama $^{6} \cdot$ Kosaku Nitta $^{7}$. Tatsuo Tsukamoto ${ }^{8}$. Shunya Uchida ${ }^{9}$ Asami Takeda ${ }^{10} \cdot$ Toshinobu Sato $^{11} \cdot$ Takashi Wada $^{12}$. Hiroki Hayashi ${ }^{13}$. Yasuhiro Akai ${ }^{14} \cdot$ Megumu Fukunaga $^{15} \cdot$ Kazuhiko Tsuruya $^{16} \cdot$ Kosuke Masutani $^{17}$. Tsuneo Konta $^{18} \cdot$ Tatsuya Shoji $^{19}$. Takeyuki Hiramatsu ${ }^{20}$. Shunsuke Goto ${ }^{21}$. Hirofumi Tamai ${ }^{22}$. Saori Nishio ${ }^{23}$. Arimasa Shirasaki ${ }^{24} \cdot$ Kojiro Nagai $^{25}$. Kunihiro Yamagata ${ }^{26} \cdot$ Hajime Hasegawa $^{27} \cdot$ Hideo Yasuda $^{28}$. Shizunori Ichida ${ }^{29} \cdot$ Tomohiko Naruse $^{30}$. Tomoya Nishino $^{31}$. Hiroshi Sobajima ${ }^{32}$. Satoshi Tanaka ${ }^{33}$. Toshiyuki Akahori ${ }^{34}$. Takafumi Ito ${ }^{35}$. Yoshio Terada ${ }^{36}$. Ritsuko Katafuchi ${ }^{37}$. Shouichi Fujimoto ${ }^{38}$. Hirokazu Okada ${ }^{39}$. Eiji Ishimura ${ }^{40}$. Junichiro J. Kazama ${ }^{41}$. Keiju Hiromura ${ }^{42}$. Tetsushi Mimura ${ }^{43}$. Satoshi Suzuki ${ }^{44}$. Yosuke Saka ${ }^{45} \cdot$ Tadashi Sofue $^{46} \cdot$ Yusuke Suzuki $^{47}$. Yugo Shibagaki ${ }^{48} \cdot$ Kiyoki Kitagawa $^{49} \cdot$ Kunio Morozumi $^{50} \cdot$ Yoshiro Fujita $^{51} \cdot$ Makoto Mizutani $^{52}$. Takashi Shigematsu $^{53} \cdot$ Naoki Kashihara $^{54} \cdot$ Hiroshi Sato $^{55} \cdot$ Seiichi Matsuo ${ }^{4} \cdot$ Ichiei Narita $^{56} \cdot$ Yoshitaka Isaka $^{2}$

1 Health and Counseling Center, Osaka University, 1-17 Machikaneyama-cho, Toyonaka, Osaka 560-0043, Japan

2 Department of Nephrology, Osaka University Graduate School of Medicine, 2-2-D11 Yamadaoka, Suita, Osaka 565-0871, Japan

3 Nakayamadera Imai Clinic, 2-8-18 Nakayamadera, Takarazuka, Hyogo 665-0861, Japan

4 Department of Nephrology, Nagoya University Graduate School of Medicine, 65 Tsurumai-cho, Showa-ku, Nagoya, Aichi 466-8550, Japan

5 Department of Nephrology, Kanazawa Medical Univeristy School of Medicine, 1-1 Daigaku, Uchinada, Kahoku, Ishikawa 920-0293, Japan

6 Department of Nephrology, Rheumatology, Endocrinology and Metabolism, Okayama University Graduate School of Medicine, Dentistry and Pharmaceutical Sciences, 2-5-1 Shikatacho, Kita-ku, Okayama, Okayama 700-8558, Japan
7 Department of Nephrology, Tokyo Women's Medical University, 8-1 Kawada-cho, Shinjuku-ku, Tokyo 162-8666, Japan

8 Department of Nephrology and Dialysis, Kitano Hospital, Tazuke Kofukai Medical Research Institute, 2-4-20 Ogimachi, Kita-ku, Osaka, Osaka 530-8480, Japan

9 Department of Internal Medicine, Teikyo University School of Medicine, 2-11-1 Kaga, Itabashi-ku, Tokyo 173-8606, Japan

10 Kidney Disease Center, Japanese Red Cross Nagoya Daini Hospital, 2-9 Myokencho, Showa-ku, Nagoya, Aichi 466-8650, Japan

11 Department of Nephrology, JCHO Sendai Hospital, 3-16-1 Tsutsumi-machi, Aoba-ku, Sendai, Miyagi 981-8501, Japan

12 Department of Nephrology and Laboratory Medicine, Kanazawa University, 13-1 Takara-machi, Kanazawa, Ishikawa 920-8641, Japan 
13 Department of Nephrology, Fujita Health University School of Medicine, 1-98 Dengakugakubo, Kutsukakecho, Toyoake, Aichi 470-1192, Japan

14 First Department of Internal Medicine, Nara Medical University, 840 Shijocho, Kashihara, Nara 634-8522, Japan

15 Division of Nephrology, Department of Internal Medicine, Toyonaka Municipal Hospital, 4-14-1 Shibaharacho, Toyonaka, Osaka 560-8565, Japan

16 Department of Integrated Therapy for Chronic Kidney Disease, Graduate School of Medical Sciences, Kyushu University, 3-1-1 Maidashi, Higashi-ku, Fukuoka, Fukuoka 812-8582, Japan

17 Division of Nephrology and Rheumatology, Department of Internal Medicine, Faculty of Medicine, Fukuoka University, 7-45-1 Nanakuma, Jonan-ku, Fukuoka, Fukuoka 814-0180, Japan

18 Department of Cardiology, Pulmonology, and Nephrology, Yamagata University School of Medicine, 2-2 Iida-Nishi, Yamagata-shi, Yamagata, Yamagata 990-9585, Japan

19 Department of Kidney Disease and Hypertension, Osaka General Medical Center, 3-1-56 Bandaihigashi, Sumiyoshi-ku, Osaka, Osaka 558-8558, Japan

20 Department of Nephrology, Konan Kosei Hospital, 137 Omatsubara, Takayacho, Konan, Aichi 483-8704, Japan

21 Division of Nephrology and Kidney Center, Kobe University Graduate School of Medicine, 7-5-1 Kusunokicho, Cuho-ku, Kobe, Hyogo 650-0017, Japan

22 Department of Nephrology, Anjo Kosei Hospital, 28 Higashihirokute, Anjocho, Anjo, Aichi 446-8602, Japan

23 Division of Rheumatology, Endocrinology and Nephrology, Hokkaido University Graduate School of Medicine, Kita 15, Nishi 7, Kita-ku, Sapporo, Hokkaido 060-8638, Japan

24 Department of Nephrology, Ichinomiya Municipal Hospital, 2-2-22 Bunkyo, Ichinomiya, Aichi 491-8558, Japan

25 Department of Nephrology, Institute of Biomedical Sciences, Tokushima University Graduate School, 3-18-15, Kuramoto-cho, Tokushima 770-8503, Japan

26 Department of Nephrology, Faculty of Medicine, University of Tsukuba, 1-1-1 Tennodai, Tsukuba, Ibaraki 305-8575, Japan

27 Department of Nephrology and Hypertension, Saitama Medical Center, Saitama Medical University, 1981 Kamoda, Kawagoe, Saitama 350-850, Japan

28 Internal Medicine 1, Hamamatsu University School of Medicine, 1-20-1 Handayama, Higashi-ku, Hamamatsu, Shizuoka 431-3192, Japan

29 Department of Nephrology, Japanese Red Cross Nagoya Daiichi Hospital, 3-35 Michishitacho, Nakamura-ku, Nagoya, Aichi 453-8511, Japan

30 Department of Nephrology, Kasugai Municipal Hospital, 1-1-1 Takakicho, Kasugai, Aichi 486-8510, Japan

31 Department of Nephrology, Nagasaki University Hospital, 1-7-1 Sakamoto, Nagasaki, Nagasaki 852-8501, Japan
32 Department of Diabetology and Nephrology, Ogaki Municipal Hospital, 4-86 Minaminokawacho, Ogaki, Gifu 503-8502, Japan

33 Department of Nephrology, Shizuoka General Hospital, 4-27-1 Kitaando, Aoi-ku, Shizuoka, Shizuoak 420-8527, Japan

34 Department of Nephrology, Chutoen General Medical Center, 1-1 Shobugaike, Kakegawa, Shizuoka 436-8555, Japan

35 Division of Nephrology, Shimane University Hospital, 89-1 Enyacho, Izumo, Shimane 693-8501, Japan

36 Department of Endocrinology, Metabolism and Nephrology, Kochi Medical School, Kochi University, Okocho Kohasu, Nankoku, Kochi 783-8505, Japan

37 Kideny Unit, National Hospital Organization Fukuokahigashi Medical Center, 1-1-1 Chidori, Koga, Fukuoka 811-3195, Japan

38 Department of Hemovascular Medicine and Artificial Organs, Faculty of Medicine, University of Miyazaki, 5200 Kihara, Kiyotakecho, Miyazaki, Miyazaki 889-1692, Japan

39 Department of Nephrology, Saitama Medical University, 38 Morohongo, Moroyama, Iruma, Saitama 350-0495, Japan

40 Department of Nephrology, Osaka City University Graduate School of Medicine, 1-4-3 Asahimachi, Abeno-ku, Osaka 545-8585, Japan

41 Department of Nephrology and Hypertension, Fukushima Medical University School of Medicine, 1 Hikariga-oka, Fukushima-City, Fukushima 960-1295, Japan

42 Department of Nephrology and Rheumatology, Gunma University Graduate School of Medicine, 3-39-22 Showa-matchi, Maebashi, Gunma 371-8511, Japan

43 Department of Nephrology, Gifu Prefectural Tajimi Hospital, 5-161 Maebatacho, Tajimi, Gifu 507-8522, Japan

44 Department of Nephrology, Kainan Hospital, 396 Minamihonden, Maegasucho, Yatomi, Aichi 498-8502, Japan

45 Department of Nephrology, Yokkaichi Municipal Hospital, 2-2-37 Shibata, Yokkaichi, Mie 510-8567, Japan

46 Department of Cardiorenal and Cerebrovascular Medicine, Kagawa University, 1750-1 Ikenobe, Miki-cho, Kita-gun, Kagawa 761-0793, Japan

47 Division of Nephrology, Department of Internal Medicine, Juntendo University Faculty of Medicine, 2-1-1 Hongo, Bunkyo-ku, Tokyo 113-8421, Japan

48 Division of Nephrology and Hypertension, Department of Internal Medicine, St. Marianna University School of Medicine, 2-16-1 Sugao, Miyamae-ku, Kawasaki, Kanagawa 216-000, Japan

49 Division of Internal Medicine, National Hospital Organization Kanazawa Medical Center, 1-1 Shimoishibikimachi, Kanazawa, Ishikawa 920-8650, Japan

50 Department of Nephrology, Masuko Memorial Hospital, 35-28 Takegashicho, Nakamura-ku, Nagoya, Aichi 453-0016, Japan 
51 Department of Nephrology, Chubu Rosai Hospital, 1-10-6 Komei, Minato-ku, Nagoya, Aichi 455-8530, Japan

52

Department of Nephrology, Handa City Hospital, 2-29

Toyocho, Handa, Aichi 475-8599, Japan

53 Department of Nephrology, Wakayama Medical University, 811-1 Kimiidera, Wakayama-City, Wakayama 641-8509, Japan

54 Department of Nephrology and Hypertension, Kawasaki Medical School, 577 Matsushima, Kurashiki, Osakayama 701-0192, Japan
55 Department of Nephrology, Endocrinology, and Vascular Medicine, Tohoku Univeristy Gradaute School of Medicine, 1-1 Seiryo-cho, Aoba-ku, Sendai, Miyagi 980-8574, Japan

56 Division of Clinical Nephrology and Rheumatology, Kidney Research Center, Niigata University Graduate School of Medical and Dental Sciences, 757 Ichibancho, Asahimachi-dori, Chuo Ward, Niigata, Niigata 951-8510, Japan 